\title{
The emerging role of RNA modifications in the regulation of mRNA stability
}

\author{
Sung Ho Boo ${ }^{1,2}$ and Yoon Ki Kim $\mathbb{B}^{1,2}$
}

\begin{abstract}
Many studies have highlighted the importance of the tight regulation of mRNA stability in the control of gene expression. mRNA stability largely depends on the mRNA nucleotide sequence, which affects the secondary and tertiary structures of the mRNAs, and the accessibility of various RNA-binding proteins to the mRNAs. Recent advances in high-throughput RNA-sequencing techniques have resulted in the elucidation of the important roles played by mRNA modifications and mRNA nucleotide sequences in regulating mRNA stability. To date, hundreds of different RNA modifications have been characterized. Among them, several RNA modifications, including $N^{6}$-methyladenosine $\left(m^{6} A\right), N^{6}, 2^{\prime}$-O-dimethyladenosine $\left(m^{6} A m\right), 8-0 \times 0-7,8$-dihydroguanosine (8-oxoG), pseudouridine $(\Psi), 5$-methylcytidine $\left(\mathrm{m}^{5} \mathrm{C}\right)$, and $N^{4}$-acetylcytidine $\left(\mathrm{ac}^{4} \mathrm{C}\right)$, have been shown to regulate mRNA stability, consequently affecting diverse cellular and biological processes. In this review, we discuss our current understanding of the molecular mechanisms underlying the regulation of mammalian mRNA stability by various RNA modifications.
\end{abstract}

\section{Introduction}

Many recent studies have demonstrated that RNA undergoes various modifications in a manner similar to DNA. These RNA modifications play a role in many cellular and biological processes, thereby opening up an emerging research field known as epitranscriptomics ${ }^{1-7}$. According to the MODOMICS database, 170 different RNA modifications have been identified in coding and noncoding RNAs ${ }^{5,8,9}$. In certain types of modifications, the specific nucleotide sequences and positions targeted for RNA modification have been well characterized due to recent advances in specialized high-throughput RNAsequencing technologies ${ }^{10}$.

Generally, the fate of a modified transcript is determined by the coordinated actions of the following three

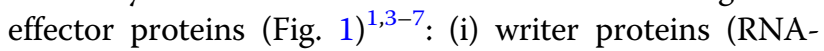
modifying enzymes), which transfer a specific chemical group to a target position on an RNA molecule; (ii) RNAbinding proteins (RBPs), which specifically recognize the

Correspondence: Yoon Ki Kim (yk-kim@korea.ac.kr)

${ }^{1}$ Creative Research Initiatives Center for Molecular Biology of Translation, Korea University, Seoul 02841, Republic of Korea

2Division of Life Sciences, Korea University, Seoul 02841, Republic of Korea modified nucleotides (reader proteins); and (iii) eraser proteins, which remove specific chemical groups from the modified nucleotides, converting them back into unmodified nucleotides. In certain cases, endogenous or exogenous chemical damage can also generate RNA modifications without the involvement of writer proteins ${ }^{11,12}$. In addition, some modifications are reversible, while others are irreversible.

These RNA modifications can affect a variety of molecular processes, such as transcription, pre-mRNA splicing, RNA export, mRNA translation, and RNA degradation ${ }^{1,3-7}$. All of these molecular events contribute to shaping the cellular transcriptome and proteome ${ }^{1-7,13}$. In particular, recent reports have posited that the regulation of mRNA stability through RNA modification is a crucial step for the tight regulation of gene expression ${ }^{1-}$ 7,13 . Therefore, in this review, we aim to highlight recent progress made in our understanding of the molecular mechanisms underlying the regulation of mRNA stability through various mRNA modifications, including $N^{6}$-methyladenosine $\left(\mathrm{m}^{6} \mathrm{~A}\right), N^{6}, 2^{\prime}$-O-dimethyladenosine $\left(\mathrm{m}^{6} \mathrm{Am}\right), \quad 8$-oxo-7,8-dihydroguanosine (8-oxoG), 


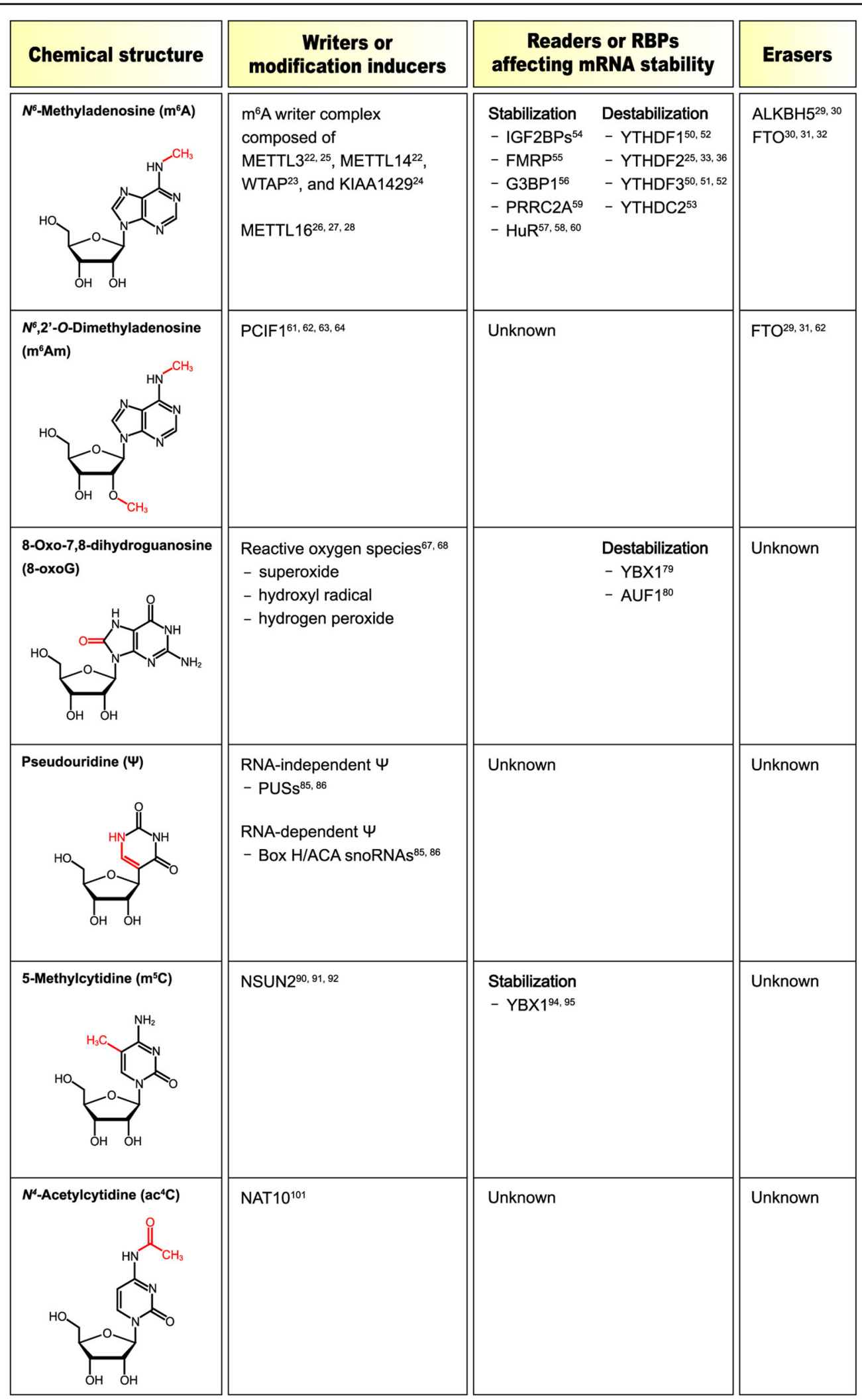

Fig. 1 Chemical structures of RNA modifications affecting mRNA stability. The chemical structures of the six RNA modifications discussed in this review are shown. The modified chemical groups are depicted in red. The known writers (or modification inducers), readers (or RBPs) involved in mRNA stability, and erasers for each RNA modification are also summarized. 
pseudouridine $(\Psi)$, 5-methylcytidine $\left(\mathrm{m}^{5} \mathrm{C}\right)$, and $N^{4}$ acetylcytidine $\left(\mathrm{ac}^{4} \mathrm{C}\right)$.

\section{$N^{6}$-methyladenosine}

$\mathrm{m}^{6} \mathrm{~A}$ is the most abundant internal mRNA modification, and it affects various cellular and physiological processes, such as maternal-to-zygotic transition (MZT), cortical neurogenesis, and the regulation of cancer stem cells in acute myeloid leukemia, ${ }^{74-19}$. A transcriptome-wide analysis for identifying the consensus sequence motifs for the $\mathrm{m}^{6} \mathrm{~A}$ modification in the human transcriptome revealed that $\mathrm{m}^{6} \mathrm{~A}$ sites $\left(\mathrm{Gm}^{6} \mathrm{AC}\right.$ or $\left.\mathrm{Am}^{6} \mathrm{AC}\right)$ are found in noncoding RNAs and mRNAs, with a greater number within long exons and adjacent to stop codons ${ }^{20,21}$.

The $\mathrm{m}^{6} \mathrm{~A}$ modification is cotranscriptionally generated in nascent transcripts by a methyltransferase complex comprising methyltransferase like 3 (METTL3),

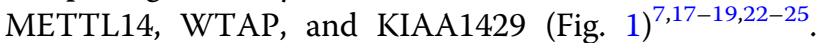
The methyltransferase complex transfers a methyl group to the N-6 position of the adenosine base. The second $\mathrm{m}^{6} \mathrm{~A}$ writer protein, METTL16 also contributes to $\mathrm{m}^{6} \mathrm{~A}$ modification of both coding and noncoding $\mathrm{RNAs}^{26-28}$. However, only a handful of mRNAs, such as MAT2A mRNA, have been identified as substrates of METTL16. $\mathrm{m}^{6} \mathrm{~A}$ is reversibly converted into adenosine by $\mathrm{m}^{6} \mathrm{~A}$ erasers (demethylases), which remove the methyl group from $m^{6} \mathrm{~A}$. The $\alpha$-ketoglutarate-dependent dioxygenase alkB homolog 5 protein (ALKBH5) is known to be the primary and specific $\mathrm{m}^{6} \mathrm{~A}$ demethylase $\mathrm{e}^{29,30}$. In addition, fat mass and obesity-associated protein (FTO) demethylase is known to have a weak preference for $\mathrm{m}^{6} \mathrm{~A}^{30-32}$.

The $\mathrm{m}^{6} \mathrm{~A}$ modification plays a regulatory role in diverse molecular processes, such as transcription, pre-mRNA splicing, mRNA export, mRNA stability, and translation $^{7,17-19}$. The molecular events that occur through the $\mathrm{m}^{6} \mathrm{~A}$ modification are guided by various $\mathrm{m}^{6} \mathrm{~A}$-recognizing reader proteins, such as YT521-B homology (YTH) domain-containing proteins. The YTHDF2 protein is the most representative $\mathrm{m}^{6} \mathrm{~A}$ reader protein involved in the decay of $\mathrm{m}^{6} \mathrm{~A}$-containing RNA (Fig. 2) ${ }^{25}$. YTHDF2 contains a $\mathrm{P} / \mathrm{Q} / \mathrm{N}$-rich unstructured region in its $\mathrm{N}$-terminal half, which is critical for YTHDF2 interactions with other cellular factors, and an RNA-binding domain in the Cterminal half, which is crucial for binding $\mathrm{m}^{6} \mathrm{~A}$-containing transcripts ${ }^{25,33-35}$. When an $\mathrm{m}^{6} \mathrm{~A}$-containing mRNA is recognized by YTHDF2, rapid degradation of mRNA is initiated in one of two distinct pathways: the deadenylation pathway or the endoribonucleolytic pathway ${ }^{19,33,36}$. Rapid deadenylation of $\mathrm{m}^{6} \mathrm{~A}$-containing mRNA is accelerated by the recruitment of a deadenylase complex (CCR4-NOT complex) ${ }^{37,38}$ to the $\mathrm{m}^{6} \mathrm{~A}$ containing mRNA via a direct interaction with the Nterminus of YTHDF2 and the SH domain of CNOT1, a component of the CCR4-NOT complex ${ }^{33}$. The resulting deadenylated $\mathrm{m}^{6} \mathrm{~A}$-containing mRNA would be more vulnerable to $3^{\prime}-5^{\prime}$ exoribonucleolytic cleavage by the exosome complex or DIS3-like enzymes ${ }^{39-43}$.

As an alternative to deadenylation followed by $3^{\prime}-5^{\prime}$ exoribonucleolytic cleavage, endoribonucleolytic cleavage of $\mathrm{m}^{6} \mathrm{~A}$-containing mRNAs can be initiated by the interplay among YTHDF2, heat-responsive protein 12 (HRSP12, also known as reactive intermediate imine deaminase A homolog), and an endoribonuclease RNase P/MRP complex (Fig. 2) ${ }^{36}$. HRSP12 has been identified as a cellular factor involved in glucocorticoid receptormediated mRNA decay ${ }^{43-46}$. The RNase P/MRP complex has been characterized as an endoribonuclease that cleaves long noncoding RNAs and mRNAs, as well as precursor forms of $5.8 \mathrm{~S}$ rRNA and tRNA ${ }^{47-49}$. When YTHDF2 binds to an $\mathrm{m}^{6} \mathrm{~A}$-containing mRNA, it recruits HRSP12, which functions as an adaptor protein that links YTHDF2 and POP1, a component of the RNase P/MRP complex $^{36}$. Results from transcriptome analyses have shown that HRSP12 preferentially binds to the GGUUC motif, typically located upstream of YTHDF2-binding sites $^{36}$. Intriguingly, HRSP12 and YTHDF2 bind to mRNA in a cooperative manner ${ }^{36}$. This cooperative interaction facilitates the efficient recruitment of the RNase P/MRP complex to the $\mathrm{m}^{6} \mathrm{~A}$-containing mRNA. Consequently, the recruited RNase P/MRP complex triggers endoribonucleolytic cleavage, mostly downstream of the YTHDF2-binding site in the $\mathrm{mRNA}^{36}$. The two resulting products, $5^{\prime}$ and $3^{\prime}$ fragments, are degraded by $3^{\prime}-5^{\prime}$ exoribonucleolytic cleavage and $5^{\prime}-3^{\prime}$ exoribonucleolytic cleavage, respectively ${ }^{39-43}$.

It should be noted that both HRSP12 and CNOT1 bind to the unstructured $\mathrm{N}$-terminal region of YTHDF2, but at different residues ${ }^{33,36}$. Amino acids $101-200$ in the $\mathrm{N}$ terminal half of YTHDF2 are required for binding to $\mathrm{CNOT}_{1}{ }^{33}$. In contrast, HRSP12 efficiently interacts with amino acids $1-100$ as well as a truncated N-terminal YTHDF2 lacking amino acids $101-200^{36}$. Therefore, the discrete binding residues in YTHDF2 can activate two distinct pathways for the decay of $\mathrm{m}^{6} \mathrm{~A}$-containing mRNA, either deadenylation by the YTHDF2-CCR4-NOT complex or endoribonucleolytic cleavage by the YTHDF2-HRSP12-RNase P/MRP complex, depending on whether HRSP12-binding sites are present in the $\mathrm{m}^{6} \mathrm{~A}$-containing mRNA. Currently, it is unknown whether components in these two pathways communicate with each other to regulate the destabilization of $\mathrm{m}^{6} \mathrm{~A}$-containing $\mathrm{mRNA}$, and which cellular environments are responsible for the preferential activation of each decay pathway.

Recent studies have shown that, in addition to YTHDF2, other YTH domain-containing proteins are also engaged in mRNA degradation. For instance, YTHDF1, YTHDF2, and YTHDF3 share a subset of target transcripts that they 


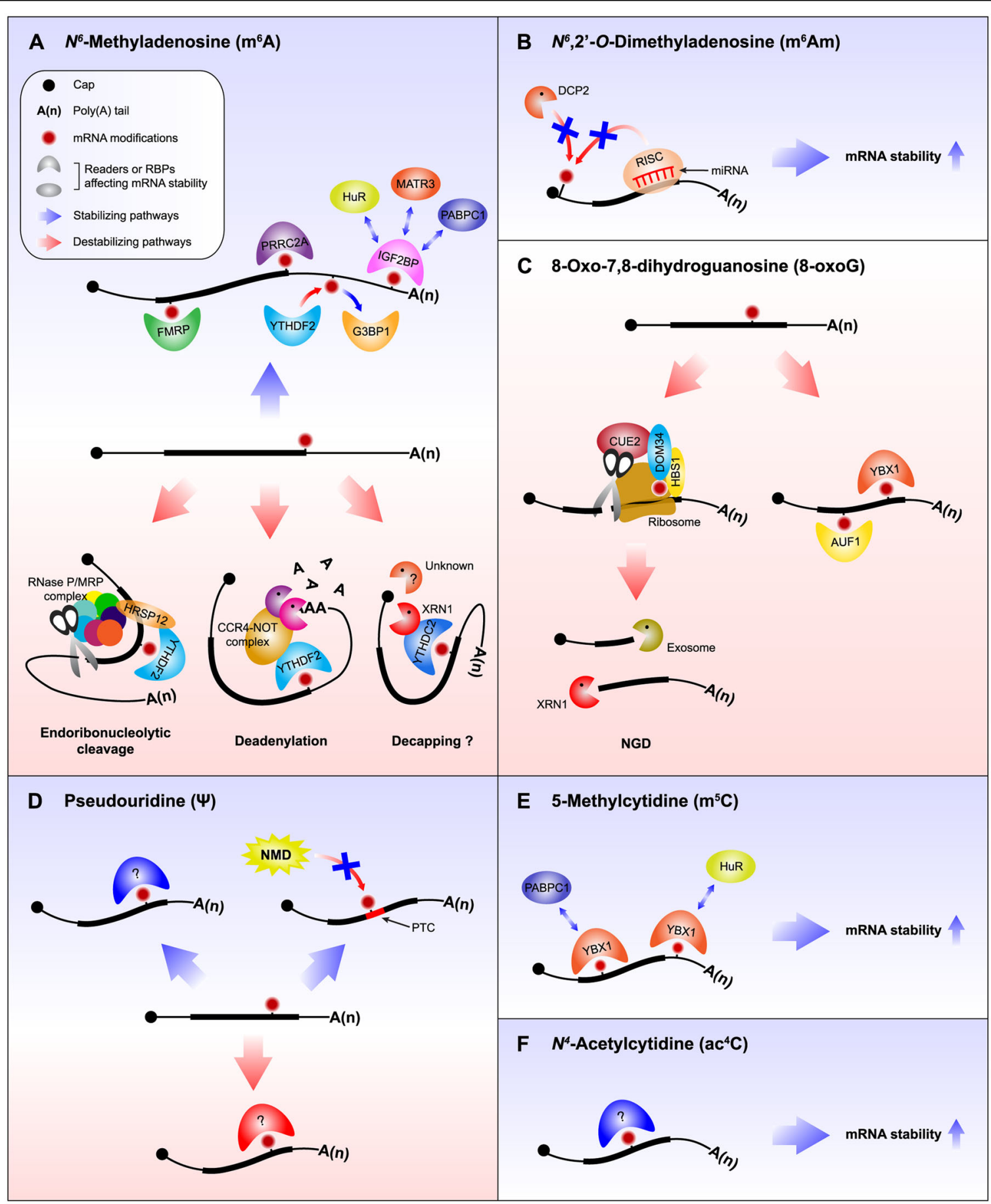

Fig. 2 Molecular mechanisms underlying the regulation of mRNA stability through diverse RNA modifications. a $N^{6}$-methyladenosine $\left(m^{6} A\right)$ : in general, YTH domain-containing proteins destabilize $\mathrm{m}^{6} \mathrm{~A}$-containing mRNAs. When $\mathrm{m}^{6} \mathrm{~A}$ is recognized by YTHDF2, the degradation of $\mathrm{m}^{6} \mathrm{~A}$ containing mRNAs is initiated by deadenylation through the CCR4-NOT complex. If the YTHDF2-bound $\mathrm{m}^{6} \mathrm{~A}$-containing mRNA harbors an HRSP12binding site, the degradation of the MRNA is preferentially initiated through an endoribonucleolytic cleavage reaction mediated by the RNase P/MRP complex. YTHDC2 binds to $\mathrm{m}^{6} \mathrm{~A}$ and recruits XRN1, thereby triggering $5^{\prime}-3^{\prime}$ exoribonucleolytic cleavage. In contrast, $\mathrm{m}^{6} \mathrm{~A}$-containing $\mathrm{mRNA}$ can be stabilized by other $\mathrm{m}^{6} \mathrm{~A}$ reader proteins or RBPs, including IGF2BP, FMRP, G3BP1, PRRC2A, and HuR. b $N^{6}, 2^{\prime}-\mathrm{O}$-dimethyladenosine ( $\left.\mathrm{m}^{6} \mathrm{Am}\right)$ : the presence of $\mathrm{m}^{6} \mathrm{Am}$ at the $5^{\prime}$-end of mRNA blocks its accessibility to DCP2, thus stabilizing the mRNA. $\mathrm{m}^{6} \mathrm{Am}$ also enables mRNA to become more resistant to microRNA-mediated mRNA degradation. c 8-Oxo-7,8-dihydroguanosine (8-oxoG): the presence of 8-oxoG in mRNA causes ribosome stalling, thereby triggering NGD. Alternatively, 8-oxoG-containing mRNAs are degraded through 8-oxoG reader proteins, such as YBX1 and AUF1. d Pseudouridine $(\Psi): \Psi$ can stabilize or destabilize mRNA. In particular, $\Psi$ on PTCs results in the inhibition of NMD. As a consequence, the mRNA is stabilized. e 5-Methylcytidine $\left(\mathrm{m}^{5} \mathrm{C}\right)$ : YBX1 specifically recognizes $\mathrm{m}^{5} \mathrm{C}$ on mRNA and recruits either PABPC1 or HuR, thereby stabilizing the mRNA. f $N^{4}$-acetylcytidine $\left(\mathrm{ac}^{4} \mathrm{C}\right)$. The presence of $\mathrm{ac}^{4} \mathrm{C}$ stabilizes mRNA by unknown mechanisms. 
destabilize ${ }^{50-52}$. In addition, the $\mathrm{N}$-terminal half of YTHDC2, another YTH domain-containing protein, interacts with XRN1, a cytoplasmic $5^{\prime}-3^{\prime}$ exoribonuclease $^{53}$, suggesting that YTHDC2 recruits XRN1 and triggers rapid degradation of $\mathrm{m}^{6} \mathrm{~A}$-containing mRNA.

$\mathrm{m}^{6} \mathrm{~A}$-modified mRNA can also be targeted toward an opposite fate, depending on the $\mathrm{m}^{6} \mathrm{~A}$ reader proteins and other RBPs (Fig. 2). For instance, a recently identified $\mathrm{m}^{6} \mathrm{~A}$ reader protein, insulin-like growth factor $2 \mathrm{mRBP}$ (IGF2BP), binds to the UGGAC motif ${ }^{54}$, which overlaps with the $\mathrm{m}^{6} \mathrm{~A}$ motif, and increases the half-life of $\mathrm{m}^{6} \mathrm{~A}$-containing mRNA $^{20,25,54}$. In addition to IGF2BP, other $\mathrm{m}^{6} \mathrm{~A}$ reader proteins or RBPs, such as fragile $\mathrm{X}$ mental retardation protein (FMRP), Ras-GTPase-activating protein SH3 domain-binding protein (G3BP1), proline-rich coiled-coil 2 A (PRRC2A), and human antigen R (HuR; also known as ELAVL1), have been shown to stabilize $\mathrm{m}^{6} \mathrm{~A}$-containing mRNA at the transcriptome or gene-specific level ${ }^{54-60}$.

\section{$N^{6}, 2^{\prime}$-O-dimethyladenosine}

The first transcribed nucleotide next to the $5^{\prime} \mathrm{m}^{7} \mathrm{G}$ cap structure in mRNA is generally methylated on the ribose ring at the $2^{\prime}-\mathrm{OH}$ position ${ }^{3-7}$. In particular, when the first nucleotide is adenosine, the methylated adenosine at the $2^{\prime}-\mathrm{OH}$ position, known as $2^{\prime}-\mathrm{O}$-methyladenosine (Am), is further methylated at the N-6 position of Am, generating $\mathrm{m}^{6} \mathrm{Am}$ (Fig. 1$)^{3-7}$. Therefore, $\mathrm{m}^{6} \mathrm{Am}$ and $\mathrm{m}^{6} \mathrm{~A}$ are generated by very similar chemical reactions: methylation at the $\mathrm{N}-6$ position of adenosine, and Am generates $\mathrm{m}^{6} \mathrm{~A}$ and $\mathrm{m}^{6} \mathrm{Am}$, respectively. However, $\mathrm{m}^{6} \mathrm{Am}$ has several properties that distinguish it from $\mathrm{m}^{6} \mathrm{~A}$. First, $\mathrm{m}^{6} \mathrm{Am}$ is generated by the methylation of $\mathrm{Am}$, which is primarily located in the first nucleotide position adjacent to the $\mathrm{m}^{7} \mathrm{G}$ cap structure of $\mathrm{mRNA}^{3-7}$. Second, a unique writer protein, phosphorylated CTD-interacting factor 1 , is responsible for generating the $\mathrm{m}^{6} \mathrm{Am}$ modification ${ }^{61-64}$, whereas a methyltransferase complex comprising METTL3, METTL14, WTAP, and KIAA1429 is involved in the generation of the $\mathrm{m}^{6} \mathrm{~A}$ modification ${ }^{22-25}$. Finally, whereas $\mathrm{m}^{6} \mathrm{~A}$ is largely demethylated by ALKBH5 but also by $\mathrm{FTO}$, with a weak preference ${ }^{29-32}, \mathrm{~m}^{6} \mathrm{Am}$ is preferentially and specifically demethylated by FTO $^{29,31,62}$.

Although controversial ${ }^{63,64}$, findings from recent studies have revealed that $\mathrm{m}^{6} \mathrm{Am}$-initiated mRNAs are in greater abundance and have longer half-lives than mRNAs with $\mathrm{Am}, \mathrm{Gm}, \mathrm{Cm}$, or $\mathrm{Um}^{31,62}$. In vitro decapping experiments have shown that $\mathrm{m}^{6} \mathrm{Am}$-initiated mRNAs are more resistant to decapping by decapping mRNA 2 (DCP2 $)^{65}$, resulting in the increased mRNA stability (Fig. 2$)^{31}$. Furthermore, $\mathrm{m}^{6} \mathrm{Am}$-initiated mRNAs are more resistant to microRNA-mediated mRNA degradation ${ }^{31}$, which also involves decapping (Fig. 2) ${ }^{66}$. Further investigation is needed to understand the molecular mechanism underlying the stabilization of $\mathrm{m}^{6} \mathrm{Am}$-initiated mRNAs.

\section{8-Oxo-7,8-dihydroguanosine}

The bases in RNA are vulnerable to various forms of chemical damage, such as those induced by reactive oxygen species (ROS), ultraviolet light, and alkylating agents $^{11}$. In particular, ROS-including superoxide, hydroxyl radicals, and hydrogen peroxide-are produced as byproducts of normal oxygen metabolism (e.g., cellular respiration in the mitochondria) and are also generated by various environmental stresses, such as ultraviolet irradiation and heat shock ${ }^{67}$. It should be noted that ROS oxidize RNA bases and generate numerous forms of oxidized RNAs ${ }^{11,67}$. These oxidized bases include 8-oxoG, 8-oxo-7,8-dihydroadenosine, 5-hydroxyuridine, 5-hydroxycytidine, and cytosine glycol (Fig. 1). Among these forms, 8-oxoG (an oxidized form of the guanine base) is the most abundant within mammalian cells, and its accumulation is associated with many neurodegenerative diseases ${ }^{68,69}$.

The oxidation of mRNA affects multiple steps of mRNA fate determination, including mRNA stability and translation $^{12,70-73}$. For instance, the oxidation of mRNA (typically 8-oxoG) inhibits the efficiency of peptide bond formation by $>1000$-fold, regardless of the codon posi$\operatorname{tion}^{71}$. This inhibition at the elongation step of translation causes the accumulation of stalled ribosomes ${ }^{71}$, which triggers the rapid mRNA degradation via the no-go decay (NGD) pathway, one of the mRNA surveillance pathways in eukaryotes (Fig. 2) ${ }^{41,74-76}$. The NGD pathway identifies stalled ribosomes caused by various impediments to translation elongation, such as robust secondary structures or stretches of rare codons in the mRNA ${ }^{77}$. The stalled ribosome is then disassembled from the mRNA and recycled. Concomitantly, the mRNA is rapidly degraded by the NGD pathway, with the coordinated action of HBS1 and DOM347. Recently, CUE2 was identified as an endoribonuclease that initiates the internal cleavage of NGD targets upstream of the stalled ribosome ${ }^{78}$. The resulting $5^{\prime}$ and $3^{\prime}$ fragments generated by endoribonucleolytic cleavage are degraded via the exosome complex and XRN1, respectively. In this way, NGD minimizes the production of truncated polypeptides (which are potentially detrimental to cells) from the ribosomes stalled because of the mRNA oxidation.

In addition to NGD, 8-oxoG-containing mRNAs are degraded by 8-oxoG reader proteins via unknown mechanisms (Fig. 2). Y-box binding protein 1 (YBX1) and AU-rich element RBP 1 (AUF1; also known as hnRNP D) have been shown to preferentially bind to 8-oxoG, thereby triggering the rapid degradation of 8-oxoG-containing $\mathrm{mRNAs}^{79,80}$. A recent study also identified poly $(\mathrm{rC})$ binding protein 1 (PCBP1) as an 8-oxoG reader protein. Notably, unlike AUF1, which recognizes a single 8-oxoG, the binding of PCBP1 to RNA requires two 8-oxoGs located in close proximity. In addition, the binding of 
PCBP1 to oxidized RNA is associated with apoptosisunder conditions of oxidative stress ${ }^{81}$-rather than with the rapid degradation of 8-oxoG-containing mRNAs, as has been observed in the case of AUF $1^{80}$. Several important questions regarding the molecular mechanisms of mRNA decay remain unanswered. First, how do 8oxoG reader proteins trigger the rapid mRNA degradation? Second, how do the 8 -oxoG reader proteins recruit general RNA-degrading enzymes? Third, is 8-oxoG reversibly converted into a normal guanosine base by a specific enzyme, as observed in the case of many other RNA modifications?

\section{Pseudouridine}

$\Psi$ is generated by the $\mathrm{C}-\mathrm{C}$ glycosidic isomerization of a uridine base (Fig. 1). Although $\Psi$ was first discovered in rRNA, tRNA, and small nuclear RNAs, evidence from recent transcriptome-wide analysis of $\Psi$ profiles in humans and yeast revealed that hundreds of human and yeast mRNAs contain $\Psi^{82,83}$. More recently, another transcriptome-wide profiling study identified thousands of $\Psi$ sites in human mRNAs ${ }^{84}$. The conversion of uridine into $\Psi$ is catalyzed by either RNA-independent or RNAdependent mechanisms ${ }^{85,86}$. In the RNA-independent mechanism, $\Psi$ is deposited by various $\Psi$ synthases (PUSs) with different substrate specificities, different chemical reactions, and different subcellular localizations. In contrast, the RNA-dependent mechanism is guided and catalyzed by Box H/ACA small nucleolar RNAs (snoRNAs).

The chemical properties of $\Psi$ differ from those of uridine $^{85,86}$. For instance, $\Psi$ makes the phosphodiester backbone more rigid, and the base pairing between $\Psi$ and adenine is stronger than that between uridine and adenine. Because of these properties, the presence of $\Psi$ in mRNAs can affect the local secondary structures and the protein-coding potential of the mRNA. Therefore, despite the lack of sufficient experimental evidence, it is plausible that $\Psi$ may directly or indirectly influence pre-mRNA splicing, mRNA translation, mRNA localization, and/or mRNA stability. Indeed, the artificially targeted conversion of U-to- $\Psi$ in translation termination codons (UAA, UGA, and UAG) turns them into missense codons ${ }^{87}$. In particular, the U-to- $\Psi$ change at premature termination codons (PTCs) can inhibit the rapid mRNA degradation triggered by nonsense-mediated mRNA decay (NMD) ${ }^{87}$, an mRNA surveillance mechanism by which faulty (e.g., PTC containing) mRNAs are specifically recognized and removed before the production of truncated (and potentially toxic) polypeptides (Fig. 2) ${ }^{41-43}$.

Several lines of evidence support the hypothesis that $\Psi$ affects mRNA stability (Fig. 2). PUS7 deletion in yeast causes a reduction in the amount of $\Psi$-containing mRNAs $^{83}$, suggesting that $\Psi$ stabilizes mRNA. In agreement with this finding, in vitro-synthesized $\Psi$-containing mRNAs are more stable than unmodified mRNAs with identical nucleotide sequences in mammalian cells ${ }^{88}$. In contrast, another study showed that, in the eukaryotic parasite Toxoplasma gondii, the half-life of the mRNAs pseudouridylated by PUS1 is significantly increased in the PUS1 mutant ${ }^{89}$, suggesting that $\Psi$ destabilizes mRNA. Therefore, future studies should focus on elucidating the molecular mechanism underlying $\Psi$-mediated regulation of mRNA stability. In addition, it should be determined whether certain RBPs have the ability to directly recognize $\Psi$, thereby affecting the stability of $\Psi$-containing mRNAs, as observed in the case of other mRNA modifications.

\section{5-Methylcytidine}

$\mathrm{m}^{5} \mathrm{C}$ is generated in transcripts by NOP2/Sun RNA methyltransferase 2 (NSUN2), which catalyzes the deposition of a methyl group at the 5 position of cytosine (Fig. 1$)^{90-92} \cdot \mathrm{m}^{5} \mathrm{C}$ is recognized by $\mathrm{m}^{5} \mathrm{C}$ reader proteins, such as $\mathrm{ALYREF}^{93}$ or $\mathrm{YBX}^{94,95}$. It remains unknown whether $\mathrm{m}^{5} \mathrm{C}$ is a reversible process because $\mathrm{m}^{5} \mathrm{C}$ erasers have not yet been identified. Similar to other RNA modifications, this modification is also present in mRNAs ${ }^{96}$. Bisulfite-sequencing analysis used to determine the $\mathrm{m}^{5} \mathrm{C}$ landscape in the human transcriptome has revealed that $\mathrm{m}^{5} \mathrm{C}$ sites are highly enriched in the $3^{\prime}$-UTR of mRNAs or near the translation initiation codon ${ }^{93,97-99}$.

A possible role of $\mathrm{m}^{5} \mathrm{C}$ in the regulation of mRNA stability has been previously implied (Fig. 2) ${ }^{98,100}$. Downregulation of NSUN2 causes a decrease in the amount and the half-life of $p 16^{I N K 4}$ mRNA, suggesting that NSUN2 functions as a stabilizer of $p 16^{I N K 4} \mathrm{mRNA}^{100}$. Furthermore, two recent studies have shown that YBX1 preferentially binds to $\mathrm{m}^{5} \mathrm{C}$-containing RNA through a $\pi-$ $\pi$ interaction between the target RNA and two tryptophan residues (Trp45 and Trp65) in the cold-shock domain of YBX1 $1^{94,95}$. This interaction contributes to the stabilization of $\mathrm{m}^{5} \mathrm{C}$-containing RNA, consequently affecting physiological events, such as the MZT (a reprogramming process during which maternal transcripts are eliminated and embryonic identity is established) and oncogene activation in human urothelial carcinoma of the bladder $(\mathrm{UCB})^{94,95}$. During early MZT in zebrafish, the interaction between $\mathrm{m}^{5} \mathrm{C}$ and YBX1 stabilizes a subset of maternal mRNAs by recruiting poly(A)-binding protein cytoplasmic 1 (PABPC 1$)^{95}$. Failure of this stabilization leads to early gastrulation defects in zebrafish embryos ${ }^{95}$. Another recent report also showed that, in human $\mathrm{UCB}$, a subset of oncogenic mRNAs have hypermethylated $\mathrm{m}^{5} \mathrm{C}$ sites and that the levels of these mRNAs are upregulated in an NSUN2-dependent manner ${ }^{94}$. In addition, the levels of NSUN2 and YBX1 proteins are higher in UCB than those in normal cells ${ }^{94}$. Mechanistically, YBX1 binds to and stabilizes oncogenic mRNAs with hypermethylated $\mathrm{m}^{5} \mathrm{C}$ 
sites (e.g., heparin-binding growth factor mRNA, which is critical for UCB progression and pathogenesis) by recruiting $\mathrm{HuR}$, thus indicating an essential oncogenic role of $\mathrm{m}^{5} \mathrm{C}$ in $\mathrm{UCB}^{94}$.

\section{$N^{4}$-acetylcytidine}

Recent transcriptome-wide profiling of another cytidine modification, $\mathrm{ac}^{4} \mathrm{C}$, in human cells showed that $\mathrm{ac}^{4} \mathrm{C}$ is widely distributed within noncoding RNAs and coding RNAs, with greater abundance near the translation initiation codon in mRNA (Fig. 1) ${ }^{101}$. mRNAs modified by $\mathrm{ac}^{4} \mathrm{C}$ are known to have increased half-lives and promoted translation (Fig. 2) ${ }^{101}$. Knocking out $N$-acetyltransferase 10 (NAT10) reduces the level of $\mathrm{ac}^{4} \mathrm{C}$ modification on RNA, indicating that NAT10 is a primary $\mathrm{ac}^{4} \mathrm{C}$ writer protein (RNA cytosine acetyltransferase) ${ }^{101}$. In yeast, orphan box C/D snoRNAs specifically guide Kre33 (a yeast homolog of human NAT10) to $\mathrm{ac}^{4} \mathrm{C}$ target sites in rRNA $^{102}$, similar to the way that $\Psi$ is guided by the $\mathrm{H}$ / ACA snoRNAs ${ }^{85,86}$. However, it remains unknown whether human NAT10 also uses box C/D snoRNAs to generate $\mathrm{ac}^{4} \mathrm{C}$ on its target RNAs. To date, neither $\mathrm{ac}^{4} \mathrm{C}$ reader protein nor an active deacetylation process has been reported. It is also unknown whether $\mathrm{ac}^{4} \mathrm{C}$ modification is a reversible process. Therefore, future studies should address the molecular mechanism underlying the stabilization of $\mathrm{ac}^{4} \mathrm{C}$-containing mRNAs.

\section{Concluding remarks}

Cellular mRNA levels are determined by various quantity control pathways (such as transcription, capping, splicing, and 3'-end formation) and quality control pathways (such as NMD and NGD). All of these molecular events are mediated by diverse cis-acting elements (e.g., nucleotide sequences and secondary structures) and trans-acting factors (e.g., RBPs and noncoding RNAs). Furthermore, recent advances have been made toward understanding the roles of RNA modifications in regulating mRNA stability. Although only certain types of modifications are discussed in this review, recent studies imply that several other RNA modifications might have the ability to influence mRNA stability. For instance, 2'-Omethylation $(\mathrm{Nm})$-in which a methyl group is added to the $2^{\prime}-\mathrm{OH}$ of the ribose ring-is highly enriched in the first and second transcribed nucleotides next to the cap structure $^{2}$. The presence of $\mathrm{Nm}$ is known to increase the levels of peroxidasin mRNA ${ }^{103}$. Furthermore, in vitro decapping experiments have shown that a capped RNA with an Nm modification is resistant to hydrolysis by the decapping exoribonuclease DXO, which specifically recognizes and removes pre-mRNAs harboring a defective cap structure ${ }^{104}$. Therefore, these two recent reports suggest that Nm functions as an mRNA stabilizer. As they are oxidized, RNAs can also be alkylated upon exposure to alkylating agents that are either endogenously produced during normal metabolic processes or exogenously provided in the environment ${ }^{11}$. Bases, riboses, and the phosphate backbone of RNA are all vulnerable to alkylation because they contain oxygen and nitrogen atoms. As a result, numerous alkylated nucleosides can be generated in RNA, possibly affecting the RNA structure and/or the protein-coding potential of mRNA. A recent study even showed that alkylated mRNA is subject to rapid degradation via $\mathrm{NGD}^{12}$. Finally, the presence of internal $N^{7}$ methylguanosine $\left(\mathrm{m}^{7} \mathrm{G}\right)$ in mRNA is known to promote translation. The positive charge of internal $\mathrm{m}^{7} \mathrm{G}$ may affect the RNA secondary structure and thereby affect the mRNA stability ${ }^{105,106}$.

Future investigations should aim to determine the transcriptome profiles of all RNA modifications, extend a list of RNA modifications that affect mRNA stability, and elucidate the underlying molecular mechanisms. In addition, considering that several mRNA modifications, such as $\Psi$, oxidation, and alkylation, are associated with mRNA surveillance pathways, it will be interesting to investigate whether mRNA surveillance pathways (NMD, NGD, and no-stop decay) ${ }^{41-43,74}$ are associated with the mRNA degradation caused by other types of RNA modifications.

\section{Acknowledgements}

This work was supported by a National Research Foundation (NRF) of Korea grant funded by the Korean government (Ministry of Science, ICT and Future Planning; NRF-2015R1A3A2033665 and NRF-2018R1A5A1024261).

\section{Conflict of interest}

The authors declare that they have no conflict of interest.

\section{Publisher's note}

Springer Nature remains neutral with regard to jurisdictional claims in published maps and institutional affiliations.

Received: 5 January 2020 Revised: 10 February 2020 Accepted: 12 February 2020.

Published online: 24 March 2020

\footnotetext{
References

1. Delaunay, S. \& Frye, M. RNA modifications regulating cell fate in cancer. Nat. Cell Biol. 21, 552-559 (2019).

2. Dimitrova, D. G., Teysset, L. \& Carre, C. RNA 2'-O-methylation (Nm) modification in human diseases. Genes (Basel) 10, 117 (2019).

3. Jonkhout, N. et al. The RNA modification landscape in human disease. RNA 23, 1754-1769 (2017).

4. Kadumuri, R. V. \& Janga, S. C. Epitranscriptomic code and its alterations in human disease. Trends Mol. Med. 24, 886-903 (2018).

5. Nachtergaele, S. \& He, C. Chemical modifications in the life of an mRNA transcript. Annu. Rev. Genet. 52, 349-372 (2018).

6. Roundtree, I. A., Evans, M. E., Pan, T. \& He, C. Dynamic RNA modifications in gene expression regulation. Cell 169, 1187-1200 (2017).

7. Shi, H., Wei, J. \& He, C. Where, when, and how: context-dependent functions of RNA methylation writers, readers, and erasers. Mol. Cell 74, 640-650 (2019).

8. Helm, M. \& Motorin, Y. Detecting RNA modifications in the epitranscriptome: predict and validate. Nat. Rev. Genet 18, 275-291 (2017).
} 
9. Boccaletto, P. et al. MODOMICS: a database of RNA modification pathways. 2017 update. Nucleic Acids Res. 46, D303-D307 (2018).

10. Li, X., Xiong, X. \& Yi, C. Epitranscriptome sequencing technologies: decoding RNA modifications. Nat. Methods 14, 23-31 (2016).

11. Yan, L. L. \& Zaher, H. S. How do cells cope with RNA damage and its consequences? J. Biol. Chem. 294, 15158-15171 (2019).

12. Yan, L. L., Simms, C. L., McLoughlin, F., Vierstra, R. D. \& Zaher, H. S. Oxidation and alkylation stresses activate ribosome-quality control. Nat. Commun. 10, 5611 (2019).

13. Singh, G., Pratt, G., Yeo, G. W. \& Moore, M. J. The clothes make the mRNA past and present trends in mRNP fashion. Annu. Rev. Biochem. 84, 325-354 (2015).

14. Zhao, B. S. et al. m(6)A-dependent maternal mRNA clearance facilitates zebrafish maternal-to-zygotic transition. Nature 542, 475-478 (2017).

15. Yoon, K. J. et al. Temporal control of mammalian cortical neurogenesis by $m$ (6)A methylation. Cell 171, 877-889 e817 (2017).

16. Paris, J. et al. Targeting the RNA m(6)A reader YTHDF2 selectively compromises cancer stem cells in acute myeloid leukemia. Cell Stem Cell 25, 137-148 e136 (2019).

17. Chen, X. Y., Zhang, J. \& Zhu, J. S. The role of m(6)A RNA methylation in human cancer. Mol. Cancer 18, 103 (2019).

18. Huang, H., Weng, H. \& Chen, J. The biogenesis and precise control of RNA $m$ (6)A methylation. Trends Genet. 36, 44-52 (2020).

19. Lee, Y., Choe, J., Park, O. H. \& Kim, Y. K. Molecular mechanisms driving mRNA degradation by m(6)A modification. Trends Genet. 36, 177-188 (2020).

20. Dominissini, D. et al. Topology of the human and mouse m6A RNA methylomes revealed by m6A-seq. Nature 485, 201-206 (2012).

21. Meyer, K. D. et al. Comprehensive analysis of mRNA methylation reveals enrichment in $3^{\prime}$ UTRs and near stop codons. Cell 149 1635-1646 (2012)

22. Liu, J. et al. A METTL3-METTL14 complex mediates mammalian nuclear RNA N6-adenosine methylation. Nat. Chem. Biol. 10, $93-95$ (2014).

23. Ping, X. L. et al. Mammalian WTAP is a regulatory subunit of the RNA N6methyladenosine methyltransferase. Cell Res. 24, 177-189 (2014).

24. Schwartz, S. et al. Perturbation of m6A writers reveals two distinct classes of mRNA methylation at internal and 5' sites. Cell Rep. 8, 284-296 (2014).

25. Wang, $X$. et al. N6-methyladenosine-dependent regulation of messenger RNA stability. Nature 505, 117-120 (2014).

26. Mendel, M. et al. Methylation of structured RNA by the m(6)A writer METTL16 is essential for mouse embryonic development. Mol. Cell 71, 986-1000 e1011 (2018)

27. Nance, D. J. et al. Characterization of METTL16 as a cytoplasmic RNA binding protein. PLOS ONE 15, e0227647 (2020).

28. Pendleton, K. E. et al. The U6 snRNA m(6)A methyltransferase METTL16 regulates SAM synthetase intron retention. Cell 169, 824-835 e814 (2017).

29. Jia, G. et al. N6-methyladenosine in nuclear RNA is a major substrate of the obesity-associated FTO. Nat. Chem. Biol. 7, 885-887 (2011).

30. Zheng, G. et al. ALKBH5 is a mammalian RNA demethylase that impacts RNA metabolism and mouse fertility. Mol. Cell 49, 18-29 (2013).

31. Mauer, J. et al. Reversible methylation of $\mathrm{m}(6) \mathrm{Am}$ in the $5^{\prime}$ cap controls mRNA stability. Nature 541, 371-375 (2017).

32. Wei, J. et al. Differential $m(6) A, m(6) A m$, and $m(1) A$ demethylation mediated by FTO in the cell nucleus and cytoplasm. Mol. Cell 71, 973-985 e975 (2018).

33. $\mathrm{Du}, \mathrm{H}$. et al. YTHDF2 destabilizes $\mathrm{m}(6) \mathrm{A}$-containing RNA through direct recruitment of the CCR4-NOT deadenylase complex. Nat. Commun. 7, 12626 (2016)

34. Reijns, M. A., Alexander, R. D., Spiller, M. P. \& Beggs, J. D. A role for Q/N-rich aggregation-prone regions in P-body localization. J. Cell Sci. 121, 2463-2472 (2008)

35. Kato, M. et al. Cell-free formation of RNA granules: low complexity sequence domains form dynamic fibers within hydrogels. Cell 149, 753-767 (2012).

36. Park, O. H. et al. Endoribonucleolytic cleavage of $\mathrm{m}(6) \mathrm{A}$-containing RNAs by RNase P/MRP complex. Mol. Cell 74, 494-507 e498 (2019).

37. Collart, M. A. The Ccr4-Not complex is a key regulator of eukaryotic gene expression. Wiley Interdiscip. Rev. RNA 7, 438-454 (2016).

38. Ukleja, M., Valpuesta, J. M., Dziembowski, A. \& Cuellar, J. Beyond the known functions of the CCR4-NOT complex in gene expression regulatory mechanisms: new structural insights to unravel CCR4-NOT mRNA processing machinery. Bioessays 38, 1048-1058 (2016).

39. Schmid, M. \& Jensen, T. H. The nuclear RNA exosome and its cofactors. Adv. Exp. Med. Biol. 1203, 113-132 (2019).
40. Saramago, M., da Costa, P. J., Viegas, S. C. \& Arraiano, C. M. The implication of mRNA degradation disorders on human disease: focus on DIS3 and DIS3-like enzymes. Adv. Exp. Med. Biol. 1157, 85-98 (2019).

41. Wolin, S. L. \& Maquat, L. E. Cellular RNA surveillance in health and disease. Science 366, 822-827 (2019).

42. Kurosaki, T., Popp, M. W. \& Maquat, L. E. Quality and quantity control of gene expression by nonsense-mediated mRNA decay. Nat. Rev. Mol. Cell Biol. 20, 406-420 (2019).

43. Kim, Y. K. \& Maquat, L. E. UPFront and center in RNA decay: UPF1 in nonsense-mediated mRNA decay and beyond. RNA 25, 407-422 (2019).

44. Park, O. H. et al. Identification and molecular characterization of cellular factors required for glucocorticoid receptor-mediated mRNA decay. Genes Dev. 30, 2093-2105 (2016)

45. Park, O. H., Do, E. \& Kim, Y. K. A new function of glucocorticoid receptor: regulation of mRNA stability. BMB Rep. 48, 367-368 (2015).

46. Cho, H. et al. Glucocorticoid receptor interacts with PNRC2 in a liganddependent manner to recruit UPF1 for rapid mRNA degradation. Proc. Natl Acad. Sci. USA 112, E1540-E1549 (2015).

47. Jarrous, N. Roles of RNase P and its subunits. Trends Genet. 33, 594-603 (2017).

48. Mattijssen, S. et al. Viperin mRNA is a novel target for the human RNase MRP/ RNase P endoribonuclease. Cell Mol. Life Sci. 68, 2469-2480 (2011).

49. Jarrous, N. \& Gopalan, V. Archaeal/eukaryal RNase P: subunits, functions and RNA diversification. Nucleic Acids Res. 38, 7885-7894 (2010).

50. Lu, W. et al. N(6)-Methyladenosine-binding proteins suppress HIV-1 infectivity and viral production. J. Biol. Chem. 293, 12992-13005 (2018).

51. Shi, H. et al. YTHDF3 facilitates translation and decay of N(6)-methyladenosine-modified RNA. Cell Res. 27, 315-328 (2017).

52. Tirumuru, $\mathrm{N}$. et al. N(6)-methyladenosine of HIV-1 RNA regulates viral infection and HIV-1 Gag protein expression. Elife 5, e15528 (2016).

53. Kretschmer, J. et al. The m(6)A reader protein YTHDC2 interacts with the small ribosomal subunit and the $5^{\prime}-3^{\prime}$ exoribonuclease XRN1. RNA 24, 1339-1350 (2018).

54. Huang, $\mathrm{H}$. et al. Recognition of RNA N(6)-methyladenosine by IGF2BP proteins enhances mRNA stability and translation. Nat. Cell Biol. 20, 285-295 (2018).

55. Zhang, F. et al. Fragile $X$ mental retardation protein modulates the stability of its m6A-marked messenger RNA targets. Hum. Mol. Genet. 27, 3936-3950 (2018).

56. Edupuganti, R. R. et al. $\mathrm{N}(6)$-methyladenosine $(\mathrm{m}(6) \mathrm{A})$ recruits and repels proteins to regulate mRNA homeostasis. Nat. Struct. Mol. Biol. 24, 870-878 (2017).

57. Visvanathan, A. et al. Essential role of METTL3-mediated m(6)A modification in glioma stem-like cells maintenance and radioresistance. Oncogene 37, 522-533 (2018).

58. Wang, Y. et al. N6-methyladenosine modification destabilizes developmental regulators in embryonic stem cells. Nat. Cell Biol. 16, 191-198 (2014).

59. Wu, R. et al. A novel m(6)A reader Prrc2a controls oligodendroglial specification and myelination. Cell Res. 29, 23-41 (2019).

60. Zhang, S. et al. m(6)A demethylase ALKBH5 maintains tumorigenicity of glioblastoma stem-like cells by sustaining FOXM1 expression and cell proliferation program. Cancer Cell 31, 591-606. e596 (2017).

61. Sun, H., Zhang, M., Li, K., Bai, D. \& Yi, C. Cap-specific, terminal N(6)-methylation by a mammalian m(6)Am methyltransferase. Cell Res. 29, 80-82 (2019).

62. Boulias, K. et al. Identification of the $m(6)$ Am methyltransferase PCIF1 reveals the location and functions of $\mathrm{m}(6) \mathrm{Am}$ in the transcriptome. Mol. Cell 75, 631-643.e638 (2019)

63. Sendinc, E. et al. PCIF1 catalyzes m6Am mRNA methylation to regulate gene expression. Mol. Cell 75, 620-630.e629 (2019).

64. Akichika, S. et al. Cap-specific terminal N (6)-methylation of RNA by an RNA polymerase II-associated methyltransferase. Science 363, eaav0080. https:/ doi.org/10.1126/science.aav0080 (2019).

65. Mugridge, J. S., Coller, J. \& Gross, J. D. Structural and molecular mechanisms for the control of eukaryotic 5'-3' mRNA decay. Nat. Struct. Mol. Biol. 25 1077-1085 (2018).

66. Rehwinkel, J, Behm-Ansmant, I, Gatfield, D. \& lzaurralde, E. A crucial role for GW182 and the DCP1:DCP2 decapping complex in miRNA-mediated gene silencing. RNA 11, 1640-1647 (2005).

67. Cadet, J. \& Wagner, J. R. DNA base damage by reactive oxygen species, oxidizing agents, and UV radiation. Cold Spring Harb. Perspect. Biol. 5, a012559 (2013). 
68. Nunomura, A., Lee, H. G., Zhu, X. \& Perry, G. Consequences of RNA oxidation on protein synthesis rate and fidelity: implications for the pathophysiology of neuropsychiatric disorders. Biochem Soc. Trans. 45, 1053-1066 (2017).

69. Poulsen, H. E. et al. RNA modifications by oxidation: a novel disease mechanism? Free Radic. Biol. Med. 52, 1353-1361 (2012).

70. Jamar, N. H., Kritsiligkou, P. \& Grant, C. M. The non-stop decay mRNA surveillance pathway is required for oxidative stress tolerance. Nucleic Acids Res. 45, 6881-6893 (2017)

71. Simms, C. L., Hudson, B. H., Mosior, J. W., Rangwala, A. S. \& Zaher, H. S. An active role for the ribosome in determining the fate of oxidized mRNA. Cell Rep. 9, 1256-1264 (2014).

72. McKinlay, A., Gerard, W. \& Fields, S. Global analysis of RNA oxidation in Saccharomyces cerevisiae. Biotechniques 52, 109-111 (2012).

73. Tanaka, M., Chock, P. B. \& Stadtman, E. R. Oxidized messenger RNA induces translation errors. Proc. Natl Acad. Sci. USA 104, 66-71 (2007)

74. Ikeuchi, K., Izawa, T. \& Inada, T. Recent progress on the molecular mechanism of quality controls induced by ribosome stalling. Front. Genet. 9, 743 (2018).

75. Simms, C. L., Thomas, E. N. \& Zaher, H. S. Ribosome-based quality control of mRNA and nascent peptides. Wiley Interdiscip. Rev. RNA 8, e1366 (2017).

76. Graille, M. \& Seraphin, B. Surveillance pathways rescuing eukaryotic ribosomes lost in translation. Nat. Rev. Mol. Cell Biol. 13, 727-735 (2012).

77. Doma, M. K. \& Parker, R. Endonucleolytic cleavage of eukaryotic mRNAs with stalls in translation elongation. Nature 440, 561-564 (2006).

78. D'Orazio, K. N. et al. The endonuclease Cue2 cleaves mRNAs at stalled ribosomes during No Go Decay. Elife 8, e49117 (2019).

79. Hayakawa, $\mathrm{H}$. et al. Binding capacity of human YB-1 protein for RNA containing 8-oxoguanine. Biochemistry 41, 12739-12744 (2002).

80. Ishii, T., Hayakawa, H., Sekiguchi, T., Adachi, N. \& Sekiguchi, M. Role of Auf1 in elimination of oxidatively damaged messenger RNA in human cells. Free Radic. Biol. Med. 79, 109-116 (2015).

81. Ishii, T., Hayakawa, H., Igawa, T., Sekiguchi, T. \& Sekiguchi, M. Specific binding of PCBP1 to heavily oxidized RNA to induce cell death. Proc. Natl Acad. Sci. USA 115, 6715-6720 (2018).

82. Carlile, T. M. et al. Pseudouridine profiling reveals regulated mRNA pseudouridylation in yeast and human cells. Nature 515, 143-146 (2014).

83. Schwartz, S. et al. Transcriptome-wide mapping reveals widespread dynamicregulated pseudouridylation of ncRNA and mRNA. Cell 159, 148-162 (2014).

84. Li, X. et al. Chemical pulldown reveals dynamic pseudouridylation of the mammalian transcriptome. Nat. Chem. Biol. 11, 592-597 (2015).

85. Adachi, H., De Zoysa, M. D. \& Yu, Y. T. Post-transcriptional pseudouridylation in mRNA as well as in some major types of noncoding RNAs. Biochim Biophys. Acta Gene Regul. Mech. 1862, 230-239 (2019).

86. Penzo, M., Guerrieri, A. N., Zacchini, F., Trere, D. \& Montanaro, L. RNA pseudouridylation in physiology and medicine: for better and for worse. Genes (Basel) 8, 301 (2017).

87. Karijolich, J. \& Yu, Y. T. Converting nonsense codons into sense codons by targeted pseudouridylation. Nature 474, 395-398 (2011).
88. Kariko, K. et al. Incorporation of pseudouridine into mRNA yields superior nonimmunogenic vector with increased translational capacity and biological stability. Mol. Ther. 16, 1833-1840 (2008).

89. Nakamoto, M. A., Lovejoy, A. F., Cygan, A. M. \& Boothroyd, J. C. mRNA pseudouridylation affects RNA metabolism in the parasite Toxoplasma gondii. RNA 23, 1834-1849 (2017).

90. Hussain, S., Aleksic, J., Blanco, S., Dietmann, S. \& Frye, M. Characterizing 5methylcytosine in the mammalian epitranscriptome. Genome Biol. 14, 215 (2013).

91. Hussain, S. et al. NSun2-mediated cytosine-5 methylation of vault noncoding RNA determines its processing into regulatory small RNAs. Cell Rep. 4 255-261 (2013).

92. Khoddami, V. \& Cairns, B. R. Identification of direct targets and modified bases of RNA cytosine methyltransferases. Nat. Biotechnol. 31, 458-464 (2013).

93. Yang, X. et al. 5-Methylcytosine promotes mRNA export - NSUN2 as the methyltransferase and ALYREF as an m(5)C reader. Cell Res. 27, 606-625 (2017)

94. Chen, X. et al. 5-Methylcytosine promotes pathogenesis of bladder cancer through stabilizing mRNAs. Nat. Cell Biol. 21, 978-990 (2019).

95. Yang, $Y$. et al. RNA 5-methylcytosine facilitates the maternal-to-zygotic transition by preventing maternal mRNA decay. Mol. Cell 75, 1188-1202 e1111 (2019).

96. Motorin, Y., Lyko, F. \& Helm, M. 5-methylcytosine in RNA: detection, enzymatic formation and biological functions. Nucleic Acids Res. 38, 1415-1430 (2010).

97. Schaefer, M., Pollex, T., Hanna, K. \& Lyko, F. RNA cytosine methylation analysis by bisulfite sequencing. Nucleic Acids Res. 37, e12 (2009).

98. Squires, J. E. et al. Widespread occurrence of 5-methylcytosine in human coding and non-coding RNA. Nucleic Acids Res. 40, 5023-5033 (2012).

99. Amort, T. et al. Distinct 5-methylcytosine profiles in poly(A) RNA from mouse embryonic stem cells and brain. Genome Biol. 18, 1 (2017).

100. Zhang, $X$. et al. The tRNA methyltransferase NSun2 stabilizes p16INK(4) mRNA by methylating the $3^{\prime}$-untranslated region of p16. Nat. Commun. 3, 712 (2012).

101. Arango, D. et al. Acetylation of cytidine in mRNA promotes translation efficiency. Cell 175, 1872-1886 (2018).

102. Sharma, S. et al. Specialized box C/D snoRNPs act as antisense guides to target RNA base acetylation. PLoS Genet. 13, e1006804 (2017).

103. Elliott, B. A. et al. Modification of messenger RNA by 2'-O-methylation regulates gene expression in vivo. Nat. Commun. 10, 3401 (2019).

104. Picard-Jean, F. et al. 2'-O-methylation of the mRNA cap protects RNAs from decapping and degradation by DXO. PLOS ONE 13, e0193804 (2018).

105. Zhang, L. S. et al. Transcriptome-wide mapping of internal N(7)-methylguanosine methylome in mammalian mRNA. Mol. Cell 74, 1304-1316. e1308 (2019).

106. Malbec, L. et al. Dynamic methylome of internal mRNA N(7)-methylguanosine and its regulatory role in translation. Cell Res. 29, 927-941 (2019). 